\title{
Decoherence from internal degrees of freedom for clusters of mesoparticles: a hierarchy of master equations
}

\author{
J. C. Flores \\ Universidad de Tarapacá \\ Departamento de Física \\ Casilla 7-D, Arica \\ Chile
}

\begin{abstract}
A mesoscopic evolution equation for an ensemble of mesoparticles follows after the elimination of internal degrees of freedom. If the system is composed of a hierarchy of scales, the reduction procedure could be worked repeatedly and the characterization of this iterating method is carried-out. Namely, a prescription describing a discrete hierarchy of master equations for the density operator is obtained. Decoherence follows from the irreversible coupling of the system, defined by mesoscopic variables, to internal degree of freedom. We discuss briefly the existence of systems with the same dynamics laws at different scales. We made an explicit calculation for an ensemble of particles with internal harmonic interaction in an external anharmonic field. New conditions related to the semiclassical limit for mesoscopic systems (Wignerfunction) are conjectured.
\end{abstract}

PACS: 03.65.B ; 03.75.Fi ; 75.40.Gb. 


\section{I.- Introduction : reduction technique}

The study of models allowing a unified description of microscopic and macroscopic physical systems has a long history. The problem is related to microscopic superposition of states and its non-occurrence at macroscopic scales. Interesting responses and different proposition can be found in references [1-18] which are related to coherence destruction by different approaches to the macroscopic level. Many of these theories are related to the original ideas developed by Landau [1] respect to the high density of states for macroscopic object, and high sensibility to external perturbation. Thus, any small perturbation produces an undefined (macroscopic) state and then mixture (i.e. decoherence). Nevertheless, it is naive to think that the physics between elementary particles and macroscopic objects, for instance like to macro-molecules, can be described only for one mesoscopic theory. This is the case of the DNA-macromolecule which, in a first level, is composed of interacting atoms and finally becomes responsible for transmission of genetic information in a biological level. Other examples are some biological composites like to the hierarchical organization of tendon, bone, mollusk shell, synthetic composites $[19,20]$ and others.

The scope of this paper is the study of an iterating systematic procedure at different discrete scales of perception. The idea is simple, we start with an ensemble of elementary particles forming clusters around its mass-centers, then we eliminate the internal degrees of freedom. In this way the dynamics law for this reduced system is obtained and the procedure could be worked again to the next level.

The general assumptions to construct the hierarchy of master equations are: 
(i) The system is composed of a hierarchy of scales, or levels, where we can recognize different architectures of clusters.

(ii) There are intercorrelation between levels. Specifically, a given set of clusters make-up a cluster in the next scale.

(iii) The dynamical requirement (equations of motion), in a given scale, is depending onto the above one.

Assumption (i) and (ii) are related to geometrical aspects, and (iii) possibilities the obtention of dynamical laws from more fundamentals scales. In fact, (iii) is related to the usual belief that phenomenological laws can be explained from fundamental models.

The paper is organized as follows. In this first section, we discuss briefly the reduction method applied to an ensemble of generic systems. In section II we deal with the iterating method and finding the master equation describing the dynamics, at different discrete scales, for interacting mesoparticles. In III some examples are briefly studied and we investigate the question related to the existence of invariant-systems under reduction procedure. In section IV, we use the Wigner function to explore the semiclassical limit for the quantum evolution equation. The mesoscopic term, related to the internal degree of freedom, requires new conditions aside the usual one related to the optical 
geometric limit. Conclusions and discussions are presented in the ending section.

Now we reviewed briefly the reduction procedure [21-27] which will be used in the next section. Consider the interaction between a system $S$ and other $R$, with many degrees of freedom, and the evolution equation for the complete system

$$
\partial_{t} \rho=\mathcal{L} \rho .
$$

We assume that the Liouville-von Neumann operator is decomposed like

$$
\mathcal{L}=\mathcal{L}_{S}+\mathcal{L}_{R}+\mathcal{L}_{I}
$$

where $\mathcal{L}_{I}$ denotes the interaction term. Consider the projector operator $P$ acting onto the total density operator $\rho$ (or the space density distribution in the classical case) : $P \rho=\rho_{R}^{e} \operatorname{Tr}_{R}(\rho)$ where $\rho_{R}^{e}$ denotes the equilibrium state of the system $R$. In the classical case, the partial-trace-operation is replaced by an integral over the phase-space of $R$. As usually, the projection operator $P$ satisfies [22] :

$$
P \mathcal{L}_{R}=\mathcal{L}_{R} P=0, \quad P \mathcal{L}_{S}=\mathcal{L}_{S} P, \quad P \mathcal{L}_{I} P=0 .
$$

In this way from the evolution equation (1), for the complete system, and projecting on the space spanned by $P$ and $Q=1-P$, one obtains the equation for $S$ :

$$
\partial_{t} \rho_{S}=\mathcal{L}_{S} \rho_{S}+\operatorname{Tr}_{R} \mathcal{L}_{I} \int_{0^{-}}^{t} d \tau e^{\mathcal{L} \tau} \mathcal{L}_{I} \rho_{R}^{e} \rho_{S}(t-\tau) .
$$

To obtain the above equation the usual initial condition $\rho(0)=\rho_{R}^{e} \rho_{S}$ was assumed. Equation (4) is exact and cumbersome because the integral term is dependent on the history of $S$. Expanding to second order in the interaction term one obtains the equation

$$
\partial_{t} \rho_{S}=\mathcal{L}_{S} \rho_{S}+\int_{0^{-}}^{t} d \tau\left\langle\mathcal{L}_{I} \mathcal{L}_{I}(\tau)\right\rangle_{R} \rho_{S}(t),
$$


where $\langle\circ\rangle_{R}$ denotes the partial trace operation $\operatorname{Tr}_{R}\left(\circ \rho_{R}^{e}\right)$, and the symbol ' $\circ$ ' means an element of the space of operators. Assuming the usual memory loss property or Markov approximation :

$$
\left\langle\mathcal{L}_{I} \mathcal{L}_{I}(\tau)\right\rangle_{R}=\gamma \delta(\tau) \mathcal{L}_{I S}^{2}
$$

with $\gamma$ a positive parameter and $\mathcal{L}_{I S}$ an operator acting on $\rho_{S}$, then we obtain from (5) and (6), the evolution equation for $S$

$$
\partial_{t} \rho_{S}=\mathcal{L}_{S} \rho_{S}+\gamma \mathcal{L}_{I S}^{2} \rho_{S}
$$

The explicit verification of the properties : $\rho_{S}=\rho_{S}^{\dagger}, \operatorname{Tr} \rho_{S}=1$ and $\rho_{S}>0$ (positivity) must be carried-out always. Remark that currently an equation like (7) is related to decoherence. Specifically, the reservoir $R$ changes any system pure states to mixed. An approximated case, where $\mathcal{L}_{I S}=\mathcal{L}_{S}$, with decoherence and without dissipation can be found in $[8,9]$. For a criticism to the reduction procedure see for instance $[10,12]$ where a completely integrable system was considered. Dissipating effects are treated in [4] (non-linear equation) where other techniques were considered.

\section{II.- Reduction procedure: hierarchiy of mas- ter equations}

In this section we use the reduction procedure sketched in section I, including a coordinate change to the center of mass, and we obtain the evolution operator at the next scale. Namely, we formalize the procedure $\mathcal{L}^{(n)} \rightarrow \mathcal{L}^{(n+1)}$. So at scale $n$, we start with $N^{(n)}$ interacting particles in an external field. This set contains an architecture of $N^{(n+1)}$ clusters which are 
recognized by using physical constraints (assumption (i)). For instance they could be $N^{(n+1)}$ molecules in an electric field or interacting macromolecules. After the cluster recognition, we consider a coordinate change to the center of mass (assumption (ii)), eliminating the internal degrees of freedom for every cluster (tracing-out-technique).

Formally at scale $n$, the equation of motion for the density distribution in the classical case, or the density operator in the quantum case, is

$$
\partial_{t} \rho^{(n)}=\mathcal{L}^{(n)} \rho^{(n)},
$$

where $\mathcal{L}^{(n)}$ denotes a linear operator constructed, for instance, by elementary Liouvillian (or von Neumann) operators like $\mathcal{L}_{f} \circ=\{f, \circ\}$, (or $\mathcal{L}_{f} \circ=$ $(1 / i \hbar)[f, \circ])$. Where the symbol $\{\circ, \circ\}$ ( or $[\circ, \circ])$ stands for the usual Poisson brackets (commutator).

In (8), the index $n$ becomes related to the discrete scale, and the idea is to obtain the evolution equation, at next scale, by constructing the new operator $\mathcal{L}^{(n+1)}$ from the dynamics at scale $n$ (assumption (iii)). In this way, the reduction procedure has technically two steps:

(a) A coordinate change to the center-of-mass of every cluster.

(b) Elimination of internal degrees of freedom by assuming loss-memory-effects (i.e. internal complexity).

Therefore, the reduction $N^{(n)} \rightarrow N^{(n+1)}$ gives an equation similar to (8), where the Liouville-von Neumann operator is determined using steps (a) and (b). To find the new evolution operator, we assume the decomposition :

$$
\mathcal{L}^{(n)}=\mathcal{L}_{K}^{(n)}+\mathcal{L}_{V}^{(n)} .
$$


Namely, a kinetic part depending on momentum and other depending on position.

To begin, we explicitly consider the first reduction procedure because it contains all the basic ingredients for further iterations. Namely, we consider the reduction $\mathcal{L}^{(0)} \rightarrow \mathcal{L}^{(1)}$, where the index $n=0$ stands for an elementary set of interacting particles forming clusters.

Let $x_{j(\alpha)}$ be the position of the particle $j$ (integer) in the cluster $\alpha$ (integer) where $1<\alpha<N^{(1)}$. Consider the transformation to the center of mass $y_{\alpha}$, of the cluster $\alpha$, given by

$$
x_{j(\alpha)}=y_{\alpha}+r_{j(\alpha)}
$$

where $r_{j(\alpha)}$ denotes the relative distance with respect $y_{\alpha}$. Consider the interacting internal potential $U_{T}$,

$$
U_{T}=\sum q_{j(\alpha)}^{k(\beta)} U\left(x_{j(\alpha)}-x_{k(\beta)}\right),
$$

where $q$ is a coupling parameter and the summation rules on all indices and no self-interactions, or repeated indices, are assumed. Moreover, like $j(\alpha)$, the term $k(\beta)$ denotes the particle $k$ in the cluster at $y_{\beta}$. From (10), the potential transforms like

$$
U_{T}=\sum q_{j(\alpha)}^{k(\beta)}\left(U\left(y_{\alpha}-y_{\beta}\right)+\left(r_{j(\alpha)}-r_{k(\beta)}\right) U^{\prime}\left(y_{\alpha}-y_{\beta}\right)\right)+F(r)
$$

where a first order multipolar expansion, in the internal coordinates, was assumed and the symbol $U^{\prime}$ denotes the first derivative. Moreover in (12), $F(r)$ denotes the linear terms depending only on the relative coordinate and related to internal interaction in the cluster. Now we define the coupling parameter, between clusters, $Q_{\alpha}^{\beta}$ and the moment $d_{\alpha, \beta}$ like

$$
Q_{\alpha}^{\beta}=\sum_{j, k} q_{j(\alpha)}^{k(\beta)}, \quad d_{\alpha, \beta}=\sum_{j, k} q_{j(\alpha)}^{k(\beta)}\left(r_{j(\alpha)}-r_{k(\beta)}\right) .
$$


and from equation (12) and (13) the internal interacting potential becomes

$$
U_{T}=\sum_{\alpha>\beta} Q_{\alpha}^{\beta} U\left(y_{\alpha}-y_{\beta}\right)+d_{\alpha, \beta} U^{\prime}\left(y_{\alpha}-y_{\beta}\right)+F(r) .
$$

Similarly, for an external field acting on every particle and given by

$$
V_{T}=\sum_{\alpha, j} q_{j(\alpha)} V\left(x_{j(\alpha)}\right)
$$

and defining the new coupling parameters and dipolar distribution by

$$
Q_{\alpha}=\sum_{j(\alpha)} q_{j(\alpha)}, \quad m_{\alpha}=\sum_{j(\alpha)} q_{j(\alpha)} r_{j(\alpha)},
$$

then the potential (15) can be written, at first order in the relative coordinates, as

$$
V_{T}=\sum_{\alpha} Q_{\alpha} V\left(y_{\alpha}\right)+m_{\alpha} V^{\prime}\left(y_{\alpha}\right)
$$

and like (12) the symbol $V^{\prime}$ denotes the first derivative. Remark that no term like $F(r)$ appears in this case. From $(14,17)$, and the transformation for the kinetic term which is form invariant, the complete Hamiltonian becomes

$$
H=H_{S}+H_{R}+H_{I}
$$

where the explicit form for the Hamiltonians are

$$
\begin{gathered}
H_{S}=\sum_{\alpha} \frac{p_{\alpha}^{2}}{2 \mu_{\alpha}}+Q_{\alpha} V\left(y_{\alpha}\right)+\sum_{\alpha>\beta} Q_{\alpha}^{\beta} U\left(y_{\alpha}-y_{\beta}\right) \\
H_{I}=\sum_{\alpha} V^{\prime}\left(y_{\alpha}\right) m_{\alpha}+\sum_{\alpha>\beta} U^{\prime}\left(y_{\alpha}-y_{\beta}\right) d_{\alpha, \beta}
\end{gathered}
$$

and $H_{R}$ is the contribution due only to internal coordinates $(r, \dot{r})$. In equation (19) the term $\mu_{\alpha}$ denotes the total mass of the cluster $\alpha$. Recall that it was always assumed a first multipolar order expansion in the internal coordinate. 
In this way, the Hamiltonian (18) has the structure worked in section I. Since the internal variables $d_{\alpha, \beta}$ and $m_{\alpha}$ are assumed independent and they have the loss-memory property then, from section I, the evolution operator for the ensemble of mesoparticles with position $y_{\alpha}$ is

$$
\mathcal{L}^{(1)}=\mathcal{L}^{(0)}(y, p)+\sum_{\alpha} \gamma_{\alpha}^{(1)}\left(\mathcal{L}_{I V}^{(1)}\left(y_{\alpha}\right)\right)^{2}+\sum_{\alpha>\beta} \gamma_{\alpha, \beta}^{(1)}\left(\mathcal{L}_{I U}^{(1)}\left(y_{\alpha}-y_{\beta}\right)\right)^{2}
$$

where we have for every component the expressions :

$$
\begin{gathered}
\mathcal{L}^{(0)}(y, p) \circ=\left\{H_{S}, \circ\right\} \\
\left(\mathcal{L}_{I V}^{(1)}\left(y_{\alpha}\right)\right)^{2} \circ=\left\{V^{\prime}\left(y_{\alpha}\right),\left\{V^{\prime}\left(y_{\alpha}\right), \circ\right\}\right\} \\
\left(\mathcal{L}_{I U}^{(1)}\left(y_{\alpha}-y_{\beta}\right)\right)^{2} \circ=\left\{U^{\prime}\left(y_{\alpha}-y_{\beta}\right),\left\{U^{\prime}\left(y_{\alpha}-y_{\beta}\right), \circ\right\}\right\}
\end{gathered}
$$

namely a double Poisson brackets, or double commutator in the quantum case.

In (21) the parameters $\gamma$ are related to white-noise type correlations between internal variables (dipole moments) for every mesoparticle. This is the Markovian approximation where memory effects are ignored. Expression (21) gives us the evolution operator $\mathcal{L}^{(1)}$ for $N^{(1)}$ mesoparticles of coordinates $\left(y_{\alpha}, p_{\alpha}\right)$, where internal degrees of freedom were eliminated. We noticed that assumption (iii), of section I, is in accord with our deduction because the evolution operator at scale $n=1$ was deducted from this one at scale $n=0$. Similar equations for one, or two mesoparticles, were also discussed in [2830]. The idea to use internal degrees of freedom as an internal environment are also discussed in [31]. 
The above evolution operator (21) is related to the first elimination of internal degrees. Nevertheless, if we can recognize a second structure of clusters, we can eliminate new internal degrees. Evidently, this will be possible only if the Markovian approximation is valid. Let $\mathcal{L}^{(n)}\left(x_{j}, q_{j}\right)$ be the evolution operator at scale $n$, which includes kinetics and potential terms like (9), and consider a cluster recognition with center of masses at $y_{\alpha}$ where $1<\alpha<N^{(n+1)}$. Then the formal first order multipolar expansion with respect to the relative coordinates (10) is

$$
\mathcal{L}^{(n)}=\mathcal{L}^{(n)}(y, Q)+\sum_{\alpha} \frac{\partial \mathcal{L}^{(n)}(y, Q)}{\partial y_{\alpha}} d_{\alpha}+F(r)
$$

where the formal derivative stands for the first order expansion, and $d_{\alpha}$ are linear function of the internal degrees. Moreover, $Q$ denotes some re-defined coupling parameters. Then still we have a situation similar to this of section (I), and if we assume the loss memory effect then the new evolution operator for the $N^{(n+1)}$ mesoparticles will be

$$
\mathcal{L}^{(n+1)}=\mathcal{L}^{(n)}(y, Q)+\sum_{\alpha} \gamma_{\alpha}^{(n)}\left(\frac{\partial \mathcal{L}^{(n)}(y, Q)}{\partial y_{\alpha}}\right)^{2} .
$$

At this point some remarks related to the iterating equation (26) are: (a) The formal derivative stands for the first order multipolar expansion around the center of mass of every mesoparticle, or cluster. (b) Expression (26) is valid in the quantum or classical case, where $\mathcal{L}$ becomes related to a set of elementary commutators or Poisson brackets. (c) Space structure (lattice, fractal, disordered, etc.) is contained in $\mathcal{L}^{0}$. It decides the criterion for the cluster recognition. (d) Remark that the free particles case $\left(\mathcal{L}_{V}=\mathcal{L}_{U}=0\right)$ is a trivial form-invariant-example under reduction procedure. 


\section{III.- Examples}

We will now examine briefly some examples related to the reduction procedure discussed in section II. Explicitly, we consider an ensemble of harmonic interacting particles with anharmonic external fields. In fact, the structure of the evolution operator becomes invariant after some reductions (aside of some redefined coupling parameters). The search for such an invariance was also investigated in [3] for a two-parameter model of decoherence, where the equation of motion for the center of mass, is formally identical to the equation for the microscopic constituents.

Consider an ensemble of particles with harmonic interaction, in a nonlinear external field. The internal and external interaction operators are given by

$$
\begin{aligned}
\mathcal{L}_{U}^{(0)} \circ= & \sum_{i \neq j}(1 / 2)\left\{K_{i, j}\left(x_{i}-x_{j}\right)^{2}, \circ\right\} . \\
& \mathcal{L}_{V}^{(0)} \circ=\lambda \sum_{j}\left\{x_{j}^{3}, \circ\right\}
\end{aligned}
$$

where $K_{i, j}$ are positive constants and $\lambda$ a coupling parameter. The expansion around $N^{(1)}$ center of mass gives

$$
\begin{gathered}
\mathcal{L}_{U}^{(0)} \circ=\sum_{\alpha \neq \beta}(1 / 2)\left\{K_{\alpha, \beta}^{\prime}\left(y_{j(\alpha)}-y_{k(\beta)}\right)^{2}, \circ\right\}+ \\
+\sum_{\alpha \neq \beta} \sum_{j, k}\left\{K_{j(\alpha), k(\beta)}\left(y_{\alpha}-y_{\beta}\right)\left(r_{j(\alpha)}-r_{k(\beta)}\right), \circ\right\}+F(r)
\end{gathered}
$$


and for the external component

$$
\mathcal{L}_{V}^{(0)} \circ=\lambda^{\prime} \sum_{\alpha}\left\{y_{\alpha}^{3}, \circ\right\}+3 \lambda \sum_{\alpha, j}\left\{y_{\alpha}^{2} r_{j(\alpha)}, \circ\right\}
$$

where $K^{\prime}$ and $\lambda^{\prime}$ are re-defined coupling parameters. The reduction procedure (section II) leads to the evolution operator

$$
\begin{aligned}
& \mathcal{L}^{(1)} \circ=\sum_{\alpha}\left\{\frac{p_{\alpha}^{2}}{2 \mu_{\alpha}}+\lambda^{\prime} y_{\alpha}^{3}, \circ\right\}+\sum_{\alpha, \beta} K_{\alpha, \beta}^{\prime}\left\{\left(y_{\alpha}-y_{\beta}\right)^{2}, \circ\right\}+ \\
& +\sum_{\alpha, \beta} \gamma_{\alpha, \beta}^{K}\left\{\left(y_{\alpha}-y_{\beta}\right),\left\{\left(y_{\alpha}-y_{\beta}\right), \circ\right\}\right\}+\sum_{\alpha} \gamma_{\alpha}^{\lambda}\left\{y_{\alpha}^{2},\left\{y_{\alpha}^{2}, \circ\right\}\right\}
\end{aligned}
$$

which gives us the evolution equation for the set of mesoparticles.

At this point we have an interesting result, a second reduction process, makes invariant the (internal) evolution operator. In fact, the only changes are related to the redefinition of the coupling parameters and mass. The same is true for the external anharmonic term in (31), which becomes invariant after three reduction process. This seems a general fact related with the formal derivative in the expression (26) for the evolution operator. So, for systems interacting algebraically (i.e. $V, U \sim x^{n}$ ) the reduction procedure seems invariant after a number finite of steps. Namely, the laws of evolution become the same at different scales of perceptions. Nevertheless, the reduction procedure must be stopped when there is not loss-memory-effect and then this process does not can be continuously carried-out. This is the case for instance for a system with a finite number of constituent. Also, we noticed that geometric aspects must be considered at every reduction, and some interesting candidates for a such invariance are elementary excitations in fractal structures. 


\section{IV.- Wigner function and the classsical limit}

Since (21) is also valid for quantum system making the appropriate changes, it is instructive to study the semiclassical limit using the Wigner function. In fact, we shall find that the semiclassical limit needs some new conditions because the decoherence term related to the parameter $\gamma$.

The Wigner function $\rho_{w}$ defined by the Fourier transform of the density operator in coordinate representation is given by

$$
\rho_{w}(x, p, t)=\frac{1}{h} \int d \eta e^{i p \eta / \hbar} \rho(x-\eta / 2, x+\eta / 2, t) .
$$

Where $\rho(x, y, t)$ is the statistical operator in coordinate representation. Then, from equation (21) and keeping by simplicity only the external potential term, the evolution for the Wigner function becomes

$$
\begin{gathered}
\partial_{t} \rho_{w}=\left\{H_{S}, \rho_{w}\right\}+\gamma\left\{\frac{\partial V}{\partial x},\left\{\frac{\partial V}{\partial x}, \rho_{w}\right\}\right\}+ \\
+\left(\hbar^{2} / 24\right)\left(\frac{\partial^{3} V}{\partial x^{3}} \frac{\partial^{3} \rho_{w}}{\partial p^{3}}-2 \gamma \frac{\partial^{2} V}{\partial x^{2}} \frac{\partial^{4} V}{\partial x^{4}} \frac{\partial^{4} \rho_{w}}{\partial p^{4}}\right)+O\left(\hbar^{4}\right) .
\end{gathered}
$$

The first two terms are the classical operators (22-23) and the other are

related to the quantum contribution. So, the mesoscopic term related to the parameter $\gamma$ gives new quantum corrections. The usual semiclassical approximation, when $\gamma=0$, is the well-known relationship

$$
\left|\frac{\partial V}{\partial x} \frac{\partial \rho_{w}}{\partial p}\right| \gg \hbar^{2}\left|\frac{\partial^{3} V}{\partial x^{3}} \frac{\partial^{3} \rho_{w}}{\partial p^{3}}\right|
$$

and related roughly to the optical geometrical limit. It must be noted, however, a similar condition related to the mesoscopic term from (33) this con- 
dition is $(\gamma \neq 0)$

$$
\left|\left(\frac{\partial^{2} V}{\partial x^{2}}\right)^{2} \frac{\partial^{2} \rho_{w}}{\partial p^{2}}\right| \gg \hbar^{2}\left|\frac{\partial^{2} V}{\partial x^{2}} \frac{\partial^{4} V}{\partial x^{4}} \frac{\partial^{4} \rho_{w}}{\partial p^{4}}\right| .
$$

Namely, it explores even derivatives in the potential.

Finally we note that the deduction of the evolution equation (33), for the Wigner function, was obtained assuming (integration by part)

$$
\left.i \hbar \partial_{x} \rho(x-\eta, x+\eta) e^{i p \eta / \hbar}\right|_{\eta=0} ^{\eta=\infty}=0 .
$$

Namely, the vanishing of the correlation term at infinite. This requirement is not always verified, for instance, consider the states $\rho=\psi(y)^{*} \psi(x)$ where the wave function is $\psi=\sin k x$ which does not satisfied (36). Nevertheless, the contribution due to the decoherence in (21), produces a fast annulment of the off-diagonal terms in the statistical operator for short-range external potential. In this way, the condition (36) can be satisfied for times greater that the decoherence time, and given a solid support to the evolution equation (33) for the Wigner function.

\section{V.- Conclusions and discussions}

We have considered a hierarchy of master equation describing the evolution, at different scales of perception, for ensembles of mesoparticles. Explic-

itly, the equation (26) gives us formally the evolution operator $\mathcal{L}^{(n+1)}$ from 
this one at scale $n$ (assumption (iii)). Its deduction requires a systematic coordinate change to the centers of mass, defined by some physical constraints, and the elimination of internal degrees of freedom is carried-out assuming loss-memory-effects. This Markovian approximation is not always valid and then, in such a case, the reduction process must be stopped. The first reduction procedure was carried-out explicitly for an ensemble of elementary components $(21)$

It must be noted that assumptions (i-iii), of section I, are the basis where our reduction procedure was developed. Namely, they possibility the obtention of a hierarchy of master equations at different scales of perception for cluster of mesoparticles. Some similarities between (i-iii) and those used in the architecture of complex synthetic assembles would be found in reference $[19,20]$.

On the other hand, some important points related to the deduction of the evolution equation (21) and (26) are :

(a) The first multipolar order expansion, in the interaction term, tell us that the asymptotic limit $t \rightarrow+\infty$ must be carried-out carefully at different scales [29].

(b) Decoherence effects at every discrete scale appears usually related to the reduction technique (section I). Thus, decoherence at macroscopic level is due to the internal complexity of every macroparticle. And quantum superposition is turned into statistical mixture .

(c) The search for invariant systems was carried-out explicitly for a model composed of interacting oscillators with an anharmonic term. It seems that other invariant systems could be found. 
(d) It was assumed that the internal moments, like to $d$ or $m$ in (13) and (16), are random and independent. Obviously this is not easy to prove and we have only assume that behavior. The statistical independence, between these random variables, is a simplification related with our calculations.

(e) The deduction of a general equation like (7) was carried-out assuming special initial conditions $\left(\rho(t=0)=\rho_{R}^{e} \rho_{S}\right)$. In our specific case of section II, these conditions not necessarily hold. More explicitly, the internal interaction cannot be switched-up arbitrary. It is an open problem to prove the validity of our procedure in this case.

To explore possible applications of our prescription, we can consider trends like wavematter, currently studied theoretically as well experimentally. After all, interacting atoms in external fields could be considered as mesoparticles. Specifically, with laser cooling techniques it becomes possible to cool atoms so that the quantum nature of atomic center of mass motion becomes important $[32,33]$. Also, it can be interesting note the growing interest in new mechanisms to break Anderson localization in disordered systems [34]. Particularly, there is the controversy about the possible enhancement of the localization length for interacting particles (TIP) [35]. This suggests considering the behavior of mesoparticles in disordered systems. Namely, an equation like to (21) with external random potential. After all, localization is a phenomenon related to coherence which is loss due to internal complexity for mesoparticles. A more detailed treatment of these points, and further physical applications will be given elsewhere.

\section{Acknowledgments}

This work was partially supported by grant FONDECYT 394000 4. It was made possible due to the influence which I received from the group of 
dynamical system directed by professor E. Tirapegui (FCFM).

\section{References}

[1] Landau L D and Lifshitz E M. Statistical Physics, 1980, Oxford Pergamon Press.

[2] Barchielli A Lanz L and Prosperi G M 1982 Nuovo Cimento B 7279

[3] Ghirardi G C Rimini A and Weber T 1986 Phys.Rev.D 34470

[4] Gisin N 1981 J.Phys.A:Math.Gen. 142259

[5] Sandulescu A and Scrutaru H 1987 Annals of Physics (NY) 173227

[6] Harris E G 1990 Phys.Rev.A 423685

[7] Daniel D and Milburn G J 1989 Phys.Rev.A 394628

[8] Milburn G J 1991 Phys.Rev.A 445401

[9] Flores J C 1995 Phys.Rev.A 512774

[10] Pechucas P 1994 Phys.Rev.Lett. 731060

[11] Petrov E G Teslenko V I and Goychuk I A 1994 Phys.Rev.E 493894

[12] van Kampen N G 1995 Jour.Stat.Phys.78 299

[13] Breuer H-P and Petruccione F 1995 Z.Phys.B 98139

[14] Leggett A J 1986 Direction In Condensed Matter Physics, G. Grinstein and G. Mazenko, Ed. World Scientific series Vol.1 Singapore

[15] Zurek W H 1981 Phys.Rev. D 24 1516. Dekker H 1977 Phys.Rev.A 16 2126 
[16] Vitali D Tessieri L and Grigolini P 1994 Phys.Rev.A 50967

[17] Zurek W H and Paz J P 1994 Phys.Rev.Lett. 722508

[18] Diosi L 1989 Phys.Rev.A 401165

[19] Baer E Hiltner A and Morgan R J 1992 Phys.Today 45 60. And references therein.

[20] Baer E Hiltner A and Keith H D 1987 Science 2351015

[21] Haake F 1973 Springer Tracts In Modern Physics 661

[22] Van Kampen N G 1992 Stochastic Processes In Physics And Chemistry (North Holland)

[23] Haken H 1975 Rev.Mod.Phys. 4767

[24] Nakajima S 1958 Prog.Theor.Phys. 20948

[25] Zwanzing R 1960 J.Chem.Phys. 331338

[26] Cohen-Tannoudji C Dupont-Roc J and Grynberg G 1992 Atom-Photon Interaction: Basic Processes And Applications (John Wiley and Sons, N.Y.)

[27] Louisell W H 1973 Quantum Statistical Properties Of Radiation, (Wiley, N.Y.)

[28] Kolovsky A R 1994 Europhys.Lett. 2779

[29] Flores J C 1995 Europhys.Lett. 29 653. Kolovsky A R 1995 Europhys.Lett. 29 655. Perez A 1995 Europhys.Lett. 3291

[30] Perez A 1996 Thesis UCV Casilla 4950 Valparaiso Chile

[31] Carazza B, preprint 1997 'On the spatial density matrix for the centre of mass of a one dimensional perfect gas', quant-ph/9711005. 
[32] Müller-Seydlitz T et al 1997 Phys.Rev.Lett. 78 1038. And references therein.

[33] Meekhof D M et al 1996 Phys.Rev.Lett. 761796

[34] Hilke M and Flores J C 1997 Phys.Rev.B 55 10625. And references therein.

[35] Römer R A and Schreiber M 1997 Phys.Rev.Lett. 78 515. Imry Y 1995 Europhys.Lett. 30 405. Shepelyansky D L 1994 Phys.Rev.Lett. 732607 